\title{
LA TRANSPARENCIA DE LA DEUDA SUBNACIONAL COMO MECANISMO PARA LIMITAR SU CRECIMIENTO
}

\author{
Marcela Astudillo, ${ }^{1}$ Andrés Blancas ${ }^{1}$ y Francisco Javier Fonseca Corona ${ }^{2}$
}

Fecha de recepción: 08 de septiembre de 2016. Fecha de aceptación: 15 de noviembre de 2016.

\begin{abstract}
RESUMEN
El objetivo de este artículo es demostrar que la ausencia de una obligación explícita de transparentar las cantidades de la deuda pública subnacional y de limitarla en la legislación correspondiente, propician su incremento acelerado. Para su análisis, se realizan estimaciones de dos modelos econométricos que combinan datos panel y series de tiempo, para determinar el impacto de la transparencia y de los límites normativos en el comportamiento de la deuda estatal. Se encontró que en promedio la deuda tendía a ser $28 \%$ mayor en las entidades federativas, donde no era obligatorio transparentar el nivel de endeudamiento; asimismo, se observó que en promedio la deuda fue menor en un $43 \%$ en las entidades que tenían límites explícitos de endeudamiento.
\end{abstract}

Palabras clave: deuda subnacional, corrupción, transparencia, finanzas públicas, modelos econométricos, entidades federativas.

Clasificación JEL: C32, C33, H63, H68.

\section{The Transparency of Subnational Debt as a Mechanism to Limit its Growth}

\begin{abstract}
The aim of this paper is to demonstrate the lack of an explicit transparency obligation in terms of disclosing the amount of subnational public debt and limiting it in relevant legislation, which effectively allow subnational debt to balloon. To analyze this matter, we estimate two econometric models that combine panel data and time series to determine the impact of transparency requirements and regulatory constraints on the behavior of state debt. It was found that on average, debt tended to be $28 \%$ higher in states where it was not mandatory to disclose debt levels; likewise, it was observed that on average, debt was $43 \%$ lower in states with explicit debt limits.
\end{abstract}

Key Words: Subnational debt, corruption, transparency, public finance, econometric models, states.

1

Instituto de Investigaciones Económicas de la Universidad Nacional Autónoma de México (UnAm), México. Correos electrónicos: marcelaa@unam.mx y neria@unam.mx, respectivamente.

2

Facultad de Economía de la Universidad Nacional Autónoma de México (unAm), México. Correo electrónico: thureos@hotmail.com 


\section{TransparenCE DETTE INFRANATIONALE COMME UN MÉCANISME POUR CROISSANCE \\ Résumé}

Le but de cet article est démontrer que l'absence d'une obligation explicite de fournir les chiffres transparentes de la dette publique sous-national et d'en limiter avec la législation pertinente, conduisent à sáugmentation rapide. Dans le dessein de l'analyser, se réalisent des estimations avec des deux modèles économétriques qui combinent des données de panel et de séries chronologiques afin de déterminer l'impact de la transparence et de réglamentation des limites sur le comportement de la dette de l'Etat. On a constaté que, en moyenne, la dette avait tendance à être $28 \%$ plus élevé dans les entités fédératives où il n'a pas les niveau de transparence obligatoire de l'endettement; également on â noté que la dette moyenne était $43 \%$ inférieure dans les entités qui ont des limites explicite de la dette.

Mots clés: la dette sous-national, la corruption, la transparence, les finances publiques, les modêles économétriques, entités fédératives.

\section{A TRANSPARÊNCIA DA DÍVIDA SUB-NACIONAL COMO UM MECANISMO PARA LI- MITAR O SEU CRESCIMENTO}

\section{Resumo}

O objetivo deste artigo é demonstrar que a ausência de uma obrigação explícita de transparência nas quantidades da dívida dos governos sub-nacionais e de limitá-la na legislaçáo pertinente proporcionam o seu rápido crescimento. Para analisar este processo, realizamos estimativas dos dois modelos econométricos que combinam dados de painel e dados de séries de tempo para determinar o impacto da transparência e dos limites dados pela regulamentação sobre o comportamento da dívida estatal. Verificou-se que, em média, a dívida tende a ser $28 \%$ maior em estados onde não foi obrigatório ter transparência do nível de endividamento; também se verificou que a dívida foi em média um $43 \%$ inferior nos estados que tinham limites de endividamento explícitos.

Palavras-chave: dívida sub-nacional, corrupção, transparência, finanças públicas, modelos econométricos, estados.

建立地方政府债务透明机制以抑制债务的增加

马赛拉・阿斯图迪略，安德鲁・布兰卡斯，弗朗西斯科・丰塞卡

摘要

本论文旨在证明如果不对地方政府债务做出明确的规定并予以立法, 将导 致债务快速增长。本文采用结合了面板和时间序列数据的两种计量模型进 行分析以确定透明度和法规限制对国债的影响。分析发现没有强制债务透 明的联邦政府机构的平均债务要比其他地区高28\%。研究同时发现：对债 务限制做出明确规定的机构，平均债务水平比其他机构低43\%。

关键词：地方政府债务，腐败，透明，公共财政，经济计量模型，联邦机构 


\section{INTRODUCCIÓN ${ }^{3}$}

Un tema que en la actualidad adquiere cada vez mayor importancia en la agenda de los organismos públicos y privados, tanto nacionales como internacionales, es el de la transparencia. Su relevancia no es casual ni ocasional, pues se debe a la desilusión que ha provocado en los ciudadanos el desempeño de los Estados, que en muchas ocasiones ha generado crisis económicas y sociales que modifican negativamente la vida de la sociedad. Aunado a este hecho, también son numerosos los escándalos de corrupción que causan irritación en la ciudadanía.

En años recientes, la deuda pública subnacional mexicana ha crecido de manera acelerada como resultado de la ausencia de un control institucional. Preguntas fundamentales cuyas respuestas resultan de gran relevancia para la sociedad en el ejercicio de su derecho a saber son: ¿De qué manera afecta la obligatoriedad explícita de transparentar el nivel de endeudamiento en el crecimiento de la deuda pública subnacional? ¿Existe alguna relación entre el crecimiento de la deuda y un marco jurídico que la límite?

Se debe tomar en cuenta que la utilización de la deuda pública sólo resuelve problemas de corto plazo, ya que tarde o temprano habrá que amortizar la deuda y cubrir su servicio en detrimento de la posibilidad de financiar servicios públicos indispensables para el bienestar de la población, que es el fin último de toda gestión gubernamental.

Con esta investigación se pretende aportar elementos para resolver este problema. Se encontró que la transparencia y el marco normativo juegan un papel de vital importancia para lograr un mayor control del endeudamiento subnacional y finanzas públicas sanas.

El tema se aborda considerando, en las dos primeras secciones, la importancia de la corrupción y la transparencia en la evolución de las finanzas públicas y particularmente en la deuda subnacional. En una tercera sección, se destaca el marco jurídico de la transparencia en México. En la cuarta se analiza el comportamiento de la deuda subnacional, así como las entidades federativas que han aplicado un límite a la contratación de deuda a partir de la reforma constitucional de 2015 en materia de disciplina financiera. En la quinta sección se presentan los argumentos, resultados y evaluación de un ejercicio econométrico de datos panel y series de tiempo que mide la relación entre 
endeudamiento subnacional, transparencia y corrupción. En la última sección se destacan algunas consideraciones finales a manera de conclusiones.

\section{LA CORRUPCIÓN Y LAS FINANZAS PÚBLICAS}

En los países latinoamericanos a pesar de la importancia que se le ha dado recientemente a la transparencia y rendición de cuentas, la debilidad de estos mecanismos y del Estado de derecho ha propiciado que persistan los actos de corrupción en la administración pública, lo que es originado en gran parte por la debilidad de los mecanismos de rendición de cuentas y del Estado de derecho (Monsiváis, 2005). Cuando la opacidad se impone da lugar a que la corrupción se constituya en práctica cotidiana que daña a la economía y, en el caso de las finanzas públicas, causar déficits que en el corto plazo son cubiertos con deuda, pero que tarde o temprano deberán ser pagados por los contribuyentes.

Para Tanzi (2008) corrupción significa ausencia de un trato justo para todos por parte de los funcionarios públicos. Como señala el autor, el problema de la corrupción es muy complejo; en primer lugar, su definición es ambigua por lo que se dificulta su identificación. El tema es muy sensible porque afecta la credibilidad y prestigio de personas e instituciones. Además, la corrupción en muchas ocasiones está protegida por personas o instituciones con poder económico y/o político. Para controlar y prevenir la corrupción se necesita tanto aumentar la probabilidad de ser sorprendido como incrementar las penas y hacerlas cumplir, de otra manera, se pierde el carácter obligatorio y será solamente un tema de voluntades políticas (Merino, 2006).

La relación entre el Estado y la corrupción es un tema fundamental. Ahora bien, a lo largo del tiempo la concepción sobre corrupción ha variado. Para Weber (2005), la corrupción es deslegitimadora de personas e instituciones que se perciben involucradas en estas prácticas. Los teóricos neoliberales como Buchanan y Musgrave (1999) conciben al Estado como fuente de rentas, privilegios y favores. Para ellos es una decisión económica normal que implica el cálculo de costo-beneficio. Para otros autores la corrupción es inevitable e incluso conveniente para vencer resistencias de las burocracias nacionales. Leff (1964) y Huntington (1968) consideran que la corrupción en las sociedades en desarrollo constituye un elemento útil para activar la vida económica. Inclusive durante el periodo de la Guerra Fría, para los países ricos era preferible tener tratos con gobernantes corruptos, pero sumisos, más que con gobernantes incorruptibles, pero también más independientes (Alonso y Garciamartín, 2011). 
Dado que la corrupción afecta la composición del gasto público y distorsiona el sistema impositivo, da lugar a una mayor posibilidad de desequilibrios en las finanzas públicas, mismos que por lo regular, son cubiertos con deuda pública. Easterly (2002) encontró evidencias de que en los países donde hay mayor corrupción el déficit público respecto al Producto Interno Bruto (PIB) es mayor que en los países con menor corrupción.

Myrdal (1990) fue uno de los pioneros en estudiar los costes que tenía para el progreso de los países la tolerancia de la corrupción. En la actualidad se considera que la corrupción distorsiona la competencia al alterar los mecanismos de asignación eficiente del mercado, concentra el ingreso al incrementar el coste de acceso a los servicios sociales de los más pobres, y es utilizada por el crimen organizado (Alonso, 2011).

El ámbito de las finanzas públicas suele ser un campo propicio para la corrupción, cuando no hay transparencia ni rendición de cuentas. En cuanto a la recaudación impositiva el resultado es que el Estado percibe menos recursos de los potenciales; esto se presenta cuando, a cambio de un pago ilegal se reducen, exentan o devuelven impuestos, pues se favorecen la evasión y elusión fiscal. Casos similares también pueden presentarse en las concesiones para explotar recursos naturales o bienes propiedad de la nación. El pago por estos derechos podría generar recursos cuantiosos al Estado, pero cuando hay corrupción, las concesiones son otorgadas a bajos costos.

Por otra parte, la corrupción desincentiva la inversión y convierte en regresivo al sistema impositivo, pues son los grandes contribuyentes los que cuentan con los mejores medios para lograr una menor carga impositiva.

Desde el punto de vista de los ingresos, los factores que favorecen la corrupción son: la complejidad del sistema tributario; la excesiva discrecionalidad de algunos administradores de impuestos. Cuando la carga tributaria es alta y el salario de los funcionarios es bajo o cuando existe una burocracia excesiva (Alonso y Garciamartín, 2011) es mayor la probabilidad de corrupción. Entre los índices que refiere la Auditoría Superior de la Federación (ASF) el Indicador de Facilidad para el Pago de Impuestos 2014-2015 (ASF, 2015) ubica a México en el lugar 105 de 189 economías.

Respecto al ejercicio del gasto público, al igual que en el caso de los ingresos, si no hay transparencia en el manejo de los recursos, a menudo aparecen mecanismos de corrupción, verbigracia a través de las licitaciones. La inversión pública se ve especialmente afectada a causa de este fenómeno, pues con frecuencia se obtienen menos beneficios de los esperados.

Ejemplo de ello son las obras construidas a precios elevados y que benefician escasamente a la población; además, en muchas ocasiones se promueven 
tales inversiones mediante el pago de sobornos (Tanzi, 2008). De esta manera se aprecia que el gasto público se incrementa debido a las comisiones que reciben los funcionarios corruptos para favorecer a un determinado proveedor en la adquisición de bienes para el sector público.

De acuerdo con Tanzi (2008), la corrupción tiene efectos adversos en los países que se reflejan en la calidad de la inversión pública, los costos de transacción para emprender negocios y el nivel de deuda pública. La corrupción también eleva el coste de determinadas operaciones económicas (Alonso y Garciamartín, 2011).

El subejercicio del presupuesto también puede ser otro reducto de corrupción. En este caso los bancos reciben cuantiosos fondos provenientes de transferencias que no se ejecutan, depositándolos en cuentas que no dan intereses o cuyos intereses quedan en manos de algún funcionario.

Por otra parte, Alt y Lassen (2006) encontraron que el déficit y la acumulación de la deuda aumentan en la medida en que se incrementa la polarización de los partidos políticos, al tratar de parecer eficientes en la provisión de servicios públicos sin incrementar los impuestos, usando el lema de "compre ahora y pague después".

Resulta imposible cuantificar la corrupción tomando como base información objetiva, pues se carece de ella, por esta razón se ha buscado medirla a través de las percepciones de distintos actores sobre la frecuencia de actos de corrupción (Del Solar, 2008). Así, en la actualidad hay diversos índices que miden la corrupción de manera indirecta. Con base en estos índices se compara la percepción de corrupción en la población de diferentes países y su evolución en el tiempo (Del Solar, 2008).

El índice de percepción de corrupción más conocido es el Índice de Transparencia Internacional desarrollado por la organización internacional no-gubernamental con el mismo nombre. Según este índice, México ocupa el lugar 95 entre 168 países y el 11 entre los 22 países de América Latina. De acuerdo al índice, entre más cerca estén los países de la calificación de 100 son menos corruptos, en 2016 México obtuvo una calificación de solamente 35.

\section{LA TRANSPARENCIA: UN MECANISMO PARA ENFRENTAR LA CORRUPCIÓN}

El rol de la transparencia es clave para el control de la corrupción (Solimano, 2008), ya que puede limitar abusos de poder; no obstante, en su ausencia, la corrupción conseguirá florecer ya que tales prácticas no se conocerán ni verán 
la luz pública. Piñar (2014: 3) cita al juez Luis B. Brandais, quien sentenció metafóricamente: "La luz del sol es el mejor de los desinfectantes". Por el contrario, la opacidad es la negación de lo público (Uvalle, 2008) su resultado es el fomento de la corrupción (Del Solar, 2008).

Pero, ¿qué es la transparencia? Es el acceso fluido y oportuno de información confiable, relevante y verificable (Del Solar, 2008). La transparencia es un medio para fortalecer la publicidad de los asuntos públicos y así evitar que el poder se ejercite de un modo que favorezca intereses privados y se desvíe de sus objetivos públicos. Desde Kant (2013) se ha considerado la transparencia como un elemento esencial del derecho público. En 1948 en la Declaración Universal de los Derechos Humanos ya se reconoce el derecho a la información.

¿Cuál es la utilidad de la transparencia en una sociedad? La transparencia puede incrementar la eficiencia en la distribución de los recursos y a la vez ser un obstáculo para que los beneficios del crecimiento sean apropiados por una élite (Bellver, 2007). La transparencia puede mejorar el control de la corrupción, hace más eficiente el uso de los recursos públicos; otorga mayores herramientas de control y fiscalización a los ciudadanos (Del Solar, 2008).

Respecto a los efectos de la transparencia en la confianza de los ciudadanos en su gobierno, no hay consenso entre los tratadistas. Desde un punto de vista optimista, la transparencia puede crear una cultura de apertura de las organizaciones gubernamentales, con lo cual se incrementará la confianza de la ciudadanía (Hollyer, Rosendorff y Vreeland, 2011); según Grimmelikhuijsen et al. (2013) la transparencia es considerada un valor clave en la confianza que los ciudadanos pueden depositar en su gobierno; sin embargo, la manera en cómo la gente percibe y aprecia la transparencia gubernamental varía según los valores culturales del país. Para los escépticos, la transparencia puede producir cierta clase de confusión entre los ciudadanos; para otros, no produce efecto alguno porque existen otros determinantes de la confianza en los gobiernos que son más importantes. En estudios empíricos recientes se concluye que la transparencia tiene un efecto limitado (Grimmelikhuijsen et al., 2013; Héritier, 2003).

Cabe subrayar que no basta con la transparencia, lo deseable es que genere cambios y no solamente que sirva como un medio de control de las decisiones gubernamentales (Arellano, 2007). Como señalan Fox y Haight (2007) la transparencia, por sí sola, no resuelve las imperfecciones del Estado de derecho, se tiene que utilizar de manera estratégica para que sea un instrumento de cambio. Con solo hacer la información disponible no se prevendrá la corrupción, se requiere además de condiciones tales como responsabilidad, 
educación, medios de comunicación independientes y elecciones libres y justas. Además, es deseable que los requerimientos de transparencia sean implementados por instituciones externas, tales como, una prensa libre, pues son más efectivos comparados con los implementados por las instituciones mismas.

Si se quieren ver efectos positivos sobre el combate a la corrupción (Lindstedt y Naurin, 2010), es importante que las reformas para aumentar la transparencia estén acompañadas por las siguientes medidas: $a$ ) fortalecer la capacidad de la ciudadanía para actuar sobre la información disponible; $b$ ) establecer sanciones cuando se incurre en abuso o incumplimiento; c) que la participación de los ciudadanos sea una realidad y participen activamente en los asuntos públicos (Uvalle, 2008); d) según Cunill (2006) para que sea transparente la información debe ser accesible para que los ciudadanos la puedan utilizar fácilmente; además debe ser relevante, que responda a los diferentes intereses de los habitantes, como pueden ser el bacheo de las calles o el gasto público destinado a la educación o el destino de la deuda pública externa, temas que no interesan por igual a todos los ciudadanos.

Aunque pareciera difícil estimar el nivel de eficiencia del gasto público asociado a la mayor transparencia, se han hecho algunos trabajos al respecto. Por ejemplo Ohashi (2009) encontró en un estudio de caso empírico, en un municipio de Japón, que mejorar la transparencia es un método efectivo para reducir el costo de adquisición de bienes y servicios del sector público, lo cual se explica porque limita abusos de discreción del funcionario encargado de las compras y debilita la colusión entre los oferentes. Las prácticas de compras opacas y discrecionales reducen los incentivos de las empresas para entrar al mercado y dan lugar a una relación perversa entre los encargados de compras del gobierno y los contratistas.

La transparencia promueve mayor competencia en procesos de licitación y contratación pública (Del Solar, 2008). Es un medio de evaluar la responsabilidad del gobierno en la toma de decisiones respecto a sus ingresos y gastos; es un insumo necesario para la participación ciudadana en la elaboración del presupuesto (Bellver, 2007). En el trabajo de Benito y Bastida (2009), utilizando un enfoque comparativo entre 41 países, demuestran una relación positiva entre el balance fiscal y la transparencia, pues mientras más información presupuestaria se conozca, los gobernantes tendrán menos oportunidades de utilizar el déficit fiscal para lograr metas en beneficio propio. También Alt y Lassen (2006) demuestran que los efectos de la transparencia fiscal son positivos sobre la disminución de la deuda pública. 


\section{EL MARCO JURÍDICO DE LA TRANSPARENCIA EN MÉXICO}

En la Constitución Política de los Estados Unidos Mexicanos (CPeum) vigente, encontramos como un primer antecedente la introducción, en 1977, del principio de que el derecho a la información será garantizado por el Estado (art. $6^{\circ}$ constitucional). La transparencia, el derecho de acceso a la información y la rendición de cuentas, son conceptos cercanos, si bien tienen significados diferentes (Luna, 2013: 35).

El presente trabajo se enfoca en la transparencia como un acto unilateral en el que los organismos públicos ponen a disposición del público la información sobre el ejercio de sus facultades y atribuciones.

La tímida reforma constitucional de 1977 permaneció como una buena intención, pero no fue sino hasta el 11 de junio de 2002 que se publicó la Ley Federal de Transparencia y Acceso a la Información Pública Gubernamental (LFTAIPG), con la finalidad proveer lo necesario para garantizar el acceso de toda persona a la información en posesión de los Poderes de la Unión, los órganos constitucionales autónomos o con autonomía legal, y cualquier otra entidad federal.

Más adelante, el Constituyente Permanente aprobó una reforma al artículo $6^{\circ}$ de la Carta Magna, publicada el 20 de julio de 2007, con el fin de establecer a nivel constitucional los principios y bases para el ejercicio del derecho de acceso a la información. Posteriormente, el 7 de febrero de 2014, se publicó en el Diario Oficial de la Federación una nueva reforma constitucional por la que se estableció un organismo autónomo, garante de la transparencia, el derecho al acceso a la información pública y la protección de datos personales, con competencia para conocer de los asuntos relacionados con esas materias de cualquier ente público federal.

En las disposiciones transitorias se mandató al Congreso de la Unión a expedir una ley reglamentaria del artículo $6^{\circ}$ constitucional. Esto último aconteció con la publicación, el 4 de mayo de 2015, de la Ley General de Transparencia y Acceso a la Información Pública (Lgatip). Por una falta de técnica legislativa, se determinó que, además de la Ley General, se reformaría la Ley Federal en la materia, paso innecesario, pues en la propia Ley General pudieron haberse distribuido facultades y competencias para cada uno de los ámbitos de gobierno, incluyendo aquellas específicas para la federación. Al fin de cuentas, el Congreso emitió una nueva Ley Federal de Transparencia y Acceso a la Información Pública ya con los plazos constitucionales completamente rebasados. 
La LFTAIPG de 2002 en su momento estableció, en su artículo $7^{\circ}$, fracción IX, que la Secretaría de Hacienda debería informar "sobre la situación económica, las finanzas públicas y la deuda pública...”. En tanto, el artículo 70 de la nueva LGTAIP dispone que "en la Ley Federal y de las Entidades Federativas se contemplará que los sujetos obligados pongan a disposición del público y mantengan actualizada, en los respectivos medios electrónicos... la información... de los temas, documentos y políticas que a continuación se señalan...”, y la fracción XXII señala expresamente "La información relativa a la deuda pública, en términos de la normatividad aplicable".

Por lo que hace las legislaciones estatales en materia de transparencia y acceso a la información pública, al momento de realizar este trabajo, en ellas se establecía como información pública de oficio diversa información financiera ( v.g. la relativa a las cuentas públicas y/o estados financieros), pero en 10 de ellas no se menciona de manera explícita la información sobre la deuda pública. Como se aprecia en el cuadro 1 , de las otras 22 , en cuatro se obligaba a publicitar tal información sólo a nivel municipal pero no estatal.

Cuadro 1. Obligación de transparencia explícita sobre deuda pública*

\begin{tabular}{lcc}
\hline Entidad Federativa & Estados & Municipios \\
\hline Aguascalientes & No & No \\
Baja California & Sí & Sí \\
Baja California Sur & Sí & Sí \\
Campeche & Sí & Sí \\
Coahuila & Sí & Sí \\
Colima & No & Sí \\
Chiapas & No & No \\
Chihuahua & No & Sí \\
Distrito Federal ${ }^{* *}$ & No & No \\
Durango & Sí & Sí \\
Guanajuato & Sí & Sí \\
Guerrero & No & No \\
Hidalgo & No & No \\
Jalisco & Sí & Sí \\
México & Sí & Sí
\end{tabular}




\begin{tabular}{|c|c|c|}
\hline Entidad Federativa & Estados & Municipios \\
\hline Michoacán & No & No \\
\hline Morelos & Sí & Sí \\
\hline Nayarit & Sí & Si \\
\hline Nuevo León & No & Sí \\
\hline Oaxaca & Sí & Sí \\
\hline Puebla & No & No \\
\hline Querétaro & No & No \\
\hline Quintana Roo & Sí & Sí \\
\hline San Luis Potosí & Sí & Sí \\
\hline Sinaloa & No & Sí \\
\hline Sonora & Sí & Sí \\
\hline Tabasco & Sí & Sí \\
\hline Tamaulipas & No & No \\
\hline Tlaxcala & No & No \\
\hline Veracruz & Sí & Sí \\
\hline Yucatán & Sí & Si \\
\hline Zacatecas & Sí & Sí \\
\hline
\end{tabular}

\section{DEUDA PÚBLICA DE LOS ESTADOS Y MUNICIPIOS}

El Constituyente de 1917 estableció dos importantes disposiciones en materia de deuda pública en la Ley Fundamental Mexicana. La primera de ellas se encuentra en el artículo 73, fracción VIII, que contiene la facultad del Congreso de la Unión de dar bases sobre las cuales el Ejecutivo pueda celebrar empréstitos, aprobar los mismos, así como para reconocer y mandar pagar la deuda nacional. La segunda de esas disposiciones se encuentra entre las limitaciones que el artículo 117 establece a los Estados: la fracción VIII estableció la prohibición de que contrajeran deuda externa. Ambas disposiciones han sufrido modificaciones, y se han presentado otras reformas constitucionales 
Gráfica 1. Deuda Subnacional Total/PIBE, 1994, 2001-2015 (Porcentaje)

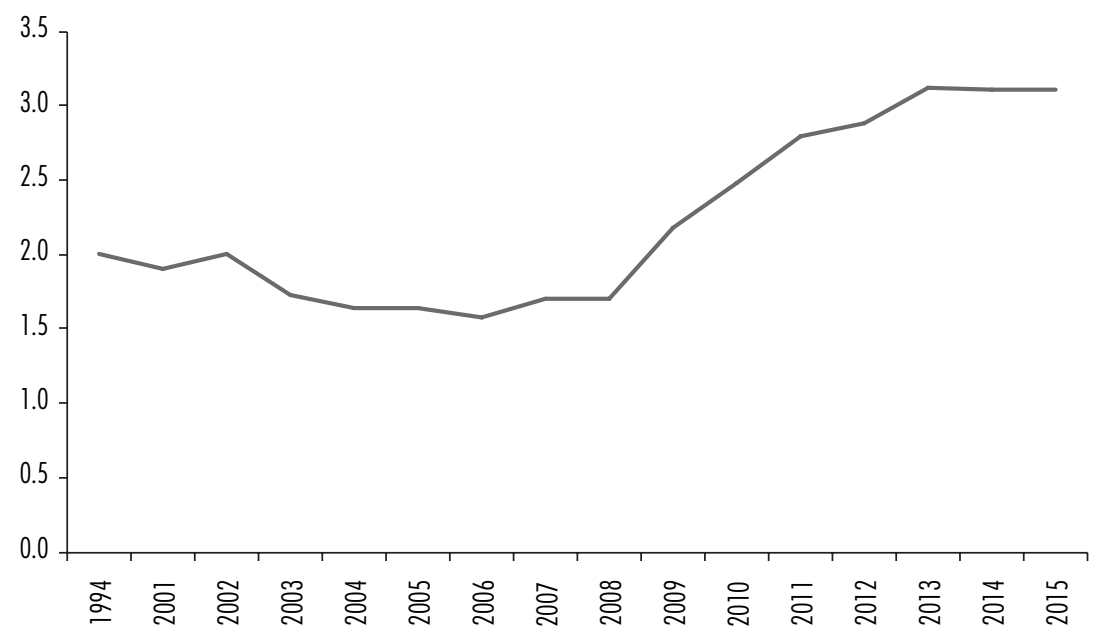

Fuente: elaborado con base en datos de la SHCP.

y a la legislación secundaria relativas a la deuda pública, en la cual se han ido incorporando candados para evitar el sobre endeudamiento de los ámbitos estatales y municipales de gobierno. Aún bajo esos límites constitucionales y legales, es notable la tendencia creciente de la deuda subnacional como proporción del Producto Interno Bruto Estatal (PIBE) en las últimas dos décadas, como puede observarse en la gráfica 1.

Recientemente se realizó una importante reforma constitucional en materia de disciplina financiera, publicada el 26 de mayo de 2015. En el artículo 73 fracción VIII, se estableció que las operaciones de refinanciamiento o reestructuración de la deuda deben realizarse bajo las mejores condiciones del mercado. Se faculta al Congreso de la Unión para expedir leyes en las que se establezcan bases sobre la deuda de las entidades federativas y los municipios, incluyendo los límites y modalidades bajo los cuales puedan afectar sus participaciones para el pago de la deuda, y la obligación de transparentar los empréstitos y obligaciones de pago a través de un registro único. Se contempla, asimismo, un sistema de alertas sobre el manejo de la deuda y la posibilidad de que hayan sanciones a los servidores públicos que incumplan con las normas en la materia. También, en caso de las entidades que tengan niveles elevados de deuda, se faculta al Congreso de la Unión para analizar, a través de una comisión legislativa bicameral, la estrategia de ajuste para fortalecer las finanzas 
públicas de aquellas que se planteen en los convenios que pretendan celebrar con el gobierno federal para obtener garantías. En el propio artículo 73 se adicionó una fracción XXIX-W en la que se faculta al Congreso de la Unión a expedir leyes en materia de responsabilidad hacendaria con el objeto de que se logre un manejo sostenible de las finanzas públicas en todos los órdenes de gobierno. Derivado de la reforma constitucional de mayo de 2015, en abril de 2016 se publicó el decreto por el que se expide la Ley de Disciplina Financiera de las Entidades Federativas y los Municipios, y se reforman, adicionan y derogan diversas disposiciones de las leyes de Coordinación Fiscal, General de Deuda Pública y General de Contabilidad Gubernamental.

El principal instrumento normativo que ha enmarcado el tema de la deuda en México ha sido la Ley General de Deuda Pública (LGDP), cuya denominación cambió, a partir del decreto mencionado a Ley Federal de Deuda Pública. La Ley, publicada el 31 de diciembre de 1976, se remonta a una época en la que el presidencialismo lograba el sometimiento de facto de los niveles subnacionales a través de facultades meta constitucionales (Carpizo, 2001: 89) y, por tanto, los límites en materia de deuda pública se fijaban más por la sumisión al titular del Ejecutivo Federal que por una norma expresa.

Así, la LGDP en realidad carecía de un elemento esencial que caracteriza a las leyes generales, que es el de distribuir competencias entre los diversos ámbitos de gobierno (Tesis: P./J. 5/2010, Semanario Judicial de la Federación y su Gaceta, Novena Época, Tomo XXXI, Febrero de 2010, pág. 2322). La LGDP más bien establece disposiciones para la administración pública descentralizada y paraestatal, las empresas de participación estatal mayoritaria, los fideicomisos en los que el fideicomitente sea el gobierno federal y las financieras de carácter público (como se constata en el cuadro 2).

De tal manera que en las carencias y lagunas jurídicas de una Ley General que en realidad nunca lo fue, podemos encontrar una parte de las causas del endeudamiento monumental en ciertos estados y municipios, y que obligó a la creación de un instrumento legal específicamente destinado a establecer criterios generales de responsabilidad hacendaria y financiera para los ámbitos subnacionales, a saber, la Ley de Disciplina Financiera de Entidades Federativas y los Municipios.

Otra parte del problema podemos hallarla, como lo veremos más adelante, en las omisiones de las leyes estatales de los estados mismas que algunos gobernadores y presidentes municipales, una vez que se vieron libres del yugo presidencialista, no dudaron en utilizar los espacios que dejaba la legislación para contratar generosos empréstitos. 
De un análisis realizado sobre las constituciones y la legislación sobre deuda de las 32 entidades federativas, se pudo encontrar que menos de la mitad de ellas contemplaba límites explícitos a la deuda de estados y municipios, entendiendo por éstos aquellos montos o porcentajes predeterminados por una disposición legal no periódica (es decir, se excluyen las leyes de ingresos y egresos que son aprobadas anualmente), ya sea en la Constitución local o una legislación secundaria, como ley de deuda, código fiscal o similar.

$\mathrm{Al}$ momento de realizarse este trabajo, de las 32 entidades federativas sólo cuatro contemplaban en su Constitución límites explícitos, y sólo una lo estableció apenas en 2014. En cuanto a la legislación secundaria, sólo en 12 de las 32 entidades había ese tipo de límites (una sola lo estableció en 2015).

De las entidades que sí contemplaban límites expresos, ya sea en la Constitución local, en la legislación secundaria o en ambas (como se observa en el cuadro 2), 11 establecen límites tanto para el estado como los municipios (reiteramos que dos de ellos lo hacen desde años recientes ya mencionados), mientras que otras cuatro sólo lo hacen para los municipios.

Cuadro 2. Límite explíito a la deuda de estados y municipios ${ }^{\star}$

\begin{tabular}{|c|c|c|c|c|}
\hline Entidad Federativa & Constitucional & Legal & Estados & Municipios \\
\hline Aguascalientes & No & Sí & Sí & Sí \\
\hline Baja California & No & Sí & Sí & Sí \\
\hline Baja California Sur & No & No & No & No \\
\hline Campeche & No & Sí & No & Sí \\
\hline Coahuila & No & No & No & No \\
\hline Colima & No & No & No & No \\
\hline Chiapas $^{* *}$ & No & Si & Sí & Sí \\
\hline Chihuahua & Sí & No & No & Sí \\
\hline Distrito Federal ${ }^{* * *}$ & $\mathrm{~N} / \mathrm{A}$ & $\mathrm{N} / \mathrm{A}$ & $\mathrm{N} / \mathrm{A}$ & $\mathrm{N} / \mathrm{A}$ \\
\hline Durango & No & Sí & № & Sí \\
\hline Guanajuato & № & No & No & No \\
\hline Guerrero & Sí & No & Sí & Sí \\
\hline Hidalgo & No & No & No & No \\
\hline Jalisco & No & Sí & Sí & Sí \\
\hline México & No & No & No & No \\
\hline
\end{tabular}




\begin{tabular}{lcccc}
\hline Entidad Federativa & Constitucional & Legal & Estados & Municipios \\
\hline Michoacán & No & Sí & Sí & Sí \\
Morelos & No & No & No & No \\
Nayarit & No & No & No & No \\
Nuevo León & No & No & No & No \\
Oaxaca & No & Sí & Sí & Sí \\
Puebla & No & No & No & No \\
Querétaro & No & No & No & No \\
Quintana Roo & No & No & No & No \\
San Luis Potosí & No & Sí & Sí & Sí \\
Sinaloa & No & No & No & No \\
Sonora & No & Sí & Sí & Sí \\
Tabasco & Sí & Sí & Sí & Sí \\
Tamaulipas & No & No & No & No \\
Tlaxcala & Sí & No & Sí & Sí \\
Veracruz & No & No & No & No \\
Yucatán & No & Sí & No & Sí \\
Zacatecas & No & No & No \\
\hline Con base & No & No & No & No \\
\hline
\end{tabular}

* Con base en la información de la página Orden Jurídico Nacional, consultada en: http://www.ordenjuridico.gob.mx/ambest.php [acceso: marzo de 2016]; ${ }^{* \star}$ Reforma de 2015; ${ }^{* \star}$ Hoy Ciudad de México. A la fecha no tiene Constitución y su deuda la aprueba el Congreso de la Unión.

Fuente: elaboración propia.

Es común también encontrar que en las legislaciones locales en materia de deuda, en muchos casos y bajo ciertas condiciones (montos y plazos), no se consideraba como deuda pública la deuda de corto plazo, lo cual es un error, pues finalmente deberá ser cubierta con dinero del erario público.

Así, podemos ver que existe una gran disparidad en los ordenamientos locales sobre deuda. No obstante, ello no significa que el problema del sobreendeudamiento haya sido solamente un problema de la estructura legal, sino más bien de la laxitud con la que algunos congresos han actuado a la hora de aprobar los montos de endeudamiento de los gobiernos subnacionales. 


\section{MODELO ECONOMÉTRICO DE TRANSPARENCIA Y LIMITACIÓN EXPLÍCITA DE DEUDA SUBNACIONAL}

A fin de hacer un acercamiento al impacto de la transparencia y en el crecimiento de la deuda pública subnacional, se presentan un par de modelos que combinan datos panel con series de tiempo que muestran la relación lineal de la deuda de cada estado de la República como porcentaje de sus participaciones federales ${ }^{4}$ con un indicador de la transparencia (si el Estado en cuestión tiene o no la obligación explícita de transparentar el nivel de la deuda) y con una proxy del marco jurídico (si el Estado en cuestión tiene un límite fijo de endeudamiento, bajo el supuesto de que el hecho de no tener un límite genera incentivos para un mayor endeudamiento). Ambos indicadores se presentan como variables dummy.

\subsection{Modelo de transparencia y deuda pública subnacional}

El modelo de transparencia y deuda pública estatal considera el logaritmo del saldo de la deuda pública estatal en proporción a los ingresos percibidos por los estados por concepto de participaciones federales, como función de la transparencia que se define como variable dummy, y se expresa en términos logarítmicos:

$\log (d e u)=c_{i}+\beta$ tra

Donde: $d e u \equiv\left(\frac{\text { dueuda estatal }}{\text { participación federal }}\right) * 100$ en un índice de participaciones. dican la presencia o ausencia de una característica, en este caso, la obligación que tiene el estado de transparentar la deuda y el límite que tiene sobre ésta. 
$c_{i} \equiv$ efecto fijo para cada estado $\mathrm{y}$;

tra $\equiv$ variable dummy sobre la transparencia de la deuda

Como variable dummy, la variable tra es igual a 1 cuando la entidad federativa tiene obligación de transparencia explícita sobre su deuda pública y es igual a 0 cuando esta obligación no existe tal y como aparece en el cuadro 1 .

El resultado es un estimador $\beta=-0.2833$, lo que significa que en promedio la deuda tiende a ser $28 \%$ mayor en las entidades federativas donde no es obligatorio transparentar el nivel de endeudamiento que en donde sí lo es.

Adicionalmente, el cuadro 4 presenta los efectos fijos por estado que capturan los aspectos que el modelo no contempla y que afectan únicamente a cada estado (constante c).

Cuadro 3. Estadísticos de la regresión

\begin{tabular}{lcccc}
\hline Variable & Coeficiente & Error Estándar & t-Stadístico & Prob. \\
\hline C & 3.767548 & 0.048715 & 77.33828 & 0.0000 \\
TRA & -0.283388 & 0.131053 & -2.162394 & 0.0312 \\
R cuadrada & 0.563365 & & & \\
\hline
\end{tabular}

Fuente: elaboración propia.

Cuadro 4. Valor del efecto fijo para cada i-Estado (valor de la constante $c_{i}$ )

\begin{tabular}{lcccc}
\hline Estado & Efecto Fijo & & Estado & Efecto Fijo \\
\hline Nacional & 0.322989 & & Nacional & 0.322989 \\
Aguascalientes & -0.144268 & & Morelos & 0.968039 \\
Baja California & 0.206752 & & Nayarit & 0.254526 \\
Baja California Sur & 0.711974 & & Nuevo León & -0.263349 \\
Campeche & 0.036776 & & Oaxaca & 0.810263 \\
Coahuila & -2.767502 & & Puebla & 1.196396 \\
Colima & -1.447889 & & Querétaro & -0.671052 \\
& & & & Continúa
\end{tabular}


Marcela Astudillo, Andrés Blancas y Francisco Javier Fonseca Corona

Cuadro 4. Valor del efecto fijo para cada i-Estado (continuación) (valor de la constante $c_{i}$ )

\begin{tabular}{lclll}
\hline Estado & Efecto Fijo & & Estado & Efecto Fijo \\
\hline Chiapas & 0.534783 & & Quintana Roo & -0.573745 \\
Chihuahua & -0.182329 & & San Luis Potosí & -0.64859 \\
Distrito Federal & -0.069839 & & Sinaloa & 0.127394 \\
Durango & -0.061676 & & Sonora & -0.144444 \\
Guanajuato & 0.605128 & & Tabasco & -0.283989 \\
Guerrero & 0.69799 & & Tamaulipas & -0.047705 \\
Hidalgo & -0.346426 & & Tlaxcala & 0.90121 \\
Jalisco & -0.133082 & & Veracruz & 0.939431 \\
México & -0.767353 & Yucatán & 0.328508 \\
Michoacán & -0.961271 & Zacatecas & 0.180476 \\
\hline
\end{tabular}

Fuente: elaboración propia.

\subsection{El impacto de la limitación explícita de la deuda pública subnacional}

El segundo modelo considera la misma variable endógena en logaritmos del modelo 1, representada por el saldo de deuda pública estatal como proporción de los ingresos estatales por concepto de participaciones federales, como una función del incremento del saldo de la deuda medida a través de una proxy sobre los límites de endeudamiento estatal ( 1 cuando existen límites normativos y 0 con no existen).

$$
\log (\text { deu }) \equiv c_{i}+c_{t}+\beta c o r
$$

Donde:

$c_{t} \equiv$ efecto fijo por cada año $\mathrm{y}$;

cor $\equiv$ proxy del incremento del saldo de la deuda

Los resultados del modelo se presentan en el cuadro 5 .

En este caso obtenemos un valor de $\beta$ igual a 0.438 , lo que nos indica que no tener límites de endeudamiento afecta directamente en el nivel de en- 
deudamiento de manera positiva. Las entidades federativas con límites de endeudamiento tienen, en promedio, $43 \%$ menos deuda que los estados sin límites. En este segundo modelo incluimos efectos propios de cada ańo (véase cuadro 6), representados por la constante $c_{t}$ en la ecuación (2), que afectan el nivel de deuda de todos los estados por igual, pero que el modelo no contempla. También se incluyen los efectos fijos de factores que no se incluyen en el modelo pero que influyen en la deuda de cada estado y están representados por la constante $c_{i}$ (véase cuadro 7). Los efectos temporales son independientes de los efectos fijos de cada estado.

Cuadro 5. Estadísticos de la regresión

\begin{tabular}{lcccc}
\hline Variable & Coeficiente & Error Estándar & t-Stadístico & Prob. \\
\hline C & 3.403798 & 0.089725 & 37.93596 & 0.0000 \\
COR & 0.438751 & 0.12677 & 3.460994 & 0.0006 \\
R cuadrada & 0.585134 & & & \\
\hline
\end{tabular}

Fuente: elaboración propia.

Cuadro 6. Efectos fijos temporales

(Valor de la constante $c_{f}$ )

\begin{tabular}{lc}
\hline Año & Efecto temporal \\
\hline 2003 & 0.040825 \\
2004 & 0.063327 \\
2005 & 0.168761 \\
2006 & 0.017697 \\
2007 & 0.025978 \\
2008 & 0.128327 \\
2009 & -0.191585 \\
2010 & -0.200206 \\
2011 & -0.053463 \\
2012 & 0.005505 \\
2013 & -0.036889 \\
2014 & 0.031725 \\
\hline
\end{tabular}

Fuente: elaboración propia. 


\begin{tabular}{|c|c|c|c|}
\hline Estado & Efecto por estado & Estado & Efecto por estado \\
\hline Nacional & 0.247987 & Nacional & 0.247987 \\
\hline Aguascalientes & 0.109794 & Morelos & 0.970719 \\
\hline Baja California & 0.428808 & Nayarit & 0.296046 \\
\hline Baja California Sur & 0.753495 & Nuevo León & -0.338351 \\
\hline Campeche & -0.038226 & Oaxaca & 0.735261 \\
\hline Coahuila & -2.815435 & Puebla & 1.121394 \\
\hline Colima & -1.52289 & Querétaro & -0.746053 \\
\hline Chiapas & 0.459782 & Quintana Roo & -0.648747 \\
\hline Chihuahua & -0.147643 & San Luis Potosí & -0.64591 \\
\hline Distrito Federal & 0.010522 & Sinaloa & 0.168915 \\
\hline Durango & 0.302073 & Sonora & -0.219446 \\
\hline Guanajuato & 0.530127 & Tabasco & -0.358991 \\
\hline Guerrero & 0.622988 & Tamaulipas & -0.122706 \\
\hline Hidalgo & -0.421428 & Tlaxcala & 0.90389 \\
\hline Jalisco & -0.169243 & Veracruz & 1.019793 \\
\hline México & -0.686992 & Yucatán & 0.370029 \\
\hline Michoacán & -0.958591 & Zacatecas & 0.105474 \\
\hline
\end{tabular}

Fuente: elaboración propia.

\subsection{Pruebas de normalidad}

Los residuos del primer modelo están distribuidos de manera normal, con una media muy cercana a cero, una asimetría también muy cercana a cero $(0.10)$ y una curtosis con un valor muy cercano a tres, ello a pesar de que el valor de la probabilidad del estadístico Jaruque-Bera es menor a 0.05 (es decir, según este estadístico no se debe aceptar la hipótesis nula de que los errores se distribuyen de manera normal, a un nivel de confianza del 95\%) (véase gráfica 2).

En el segundo modelo ocurre casi lo mismo. Los residuos del modelo tienen una distribución normal, con media cercana a cero, asimetría cercana a cero también (0.06) y una curtosis cercana a tres (véase gráfica 3). 
Gráfica 2

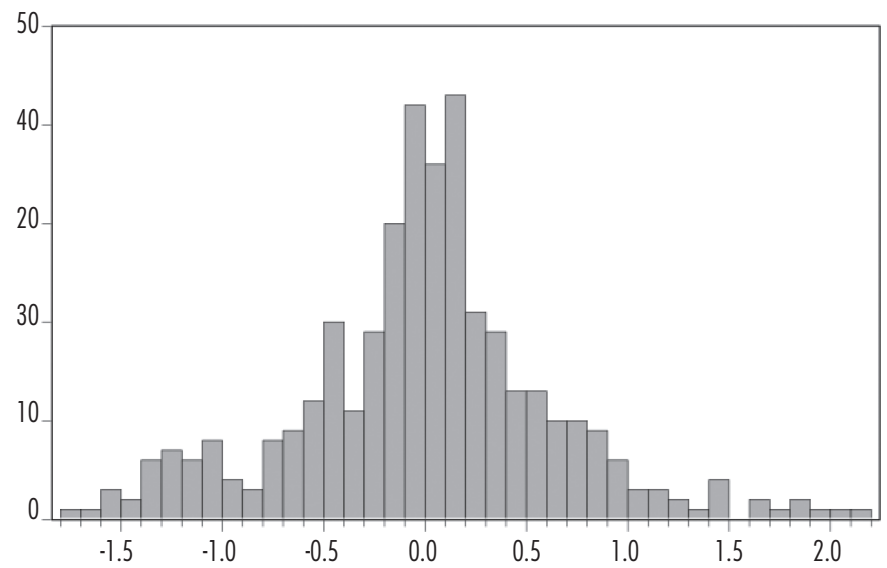

Series: Standardized Residuals Sample 20032014

Observations 393

Mean $\quad 1.58 \mathrm{e}-17$

Median $\quad 0.018121$

Maximum 2.132970

Minimum $\quad-1.787017$

Std. Dev. $\quad 0.642563$

Skewness $\quad 0.109994$

Kurtosis $\quad 3.891973$

Jarque-Bera $\quad 13.82067$

Probability $\quad 0.000997$

Fuente: elaboración propia.

\section{Gráfica 3}

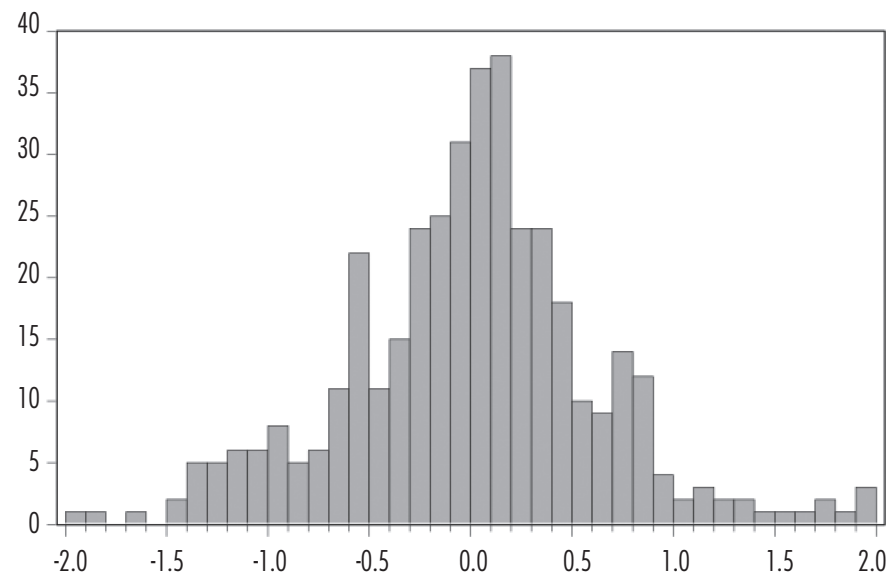

Series: Standardized Residuals Sample 20032014

Observations 393

Mean $\quad 1.13 \mathrm{e}-18$

Median $\quad 0.026204$

Maximum $\quad 1.968314$

Minimum $\quad-1.955778$

Std. Dev. $\quad 0.626340$

Skewness $\quad 0.065072$

Kurtosis $\quad 3.865038$

Jarque-Bera $\quad 12.53061$

Probability $\quad 0.001901$

Fuente: elaboración propia. 


\section{CONSIDERACIONES FINALES}

El ámbito de las finanzas públicas suele ser un campo propicio para la corrupción cuando la transparencia y el marco institucional son inexistentes o bien insuficientes. En el tema del endeudamiento resulta relevante el efecto de la corrupción, pues como se dijo anteriormente, ésta incrementa la posibilidad de déficits que por lo regular son cubiertos con deuda, puesto que la corrupción afecta la composición del gasto público y distorsiona el sistema impositivo.

Del análisis jurídico realizado se advierte que el incremento desmesurado de la deuda se presentó en el marco de una legislación desarticulada y compleja. Del análisis cuantitativo se logra el objetivo de demostrar que la ausencia de una obligación de transparencia explícita sobre la deuda pública estatal, así como de límites impuestos mediante leyes a la deuda pública de las entidades de la República, tienen repercusiones directas sobre el nivel de endeudamiento de las propias entidades federativas. El modelo demuestra con estimaciones estadísticamente significativas, que cumplen con los supuestos de normalidad la hipótesis de que una mayor transparencia en el manejo de las finanzas públicas, y específicamente una mayor obligación de transparencia explícita sobre la deuda pública subnacional, tiende a reducir el saldo de la misma; mientras que la ausencia de límites explícitos a la deuda subnacional impulsa el aumento sin control de los saldos de deuda.

A partir de los resultados obtenidos se encontró que en promedio la deuda tiende a ser $28 \%$ mayor en las entidades federativas donde no es obligatorio transparentar el nivel de endeudamiento que en las que sí lo es. Asimismo, se observó que las entidades federativas con límites explícitos de endeudamiento tienen, en promedio, 43\% menos deuda que donde no hay límites.

Si como se ha demostrado, la falta de transparencia y de una legislación adecuada tienen una incidencia en el incremento de la deuda, es necesario que el marco jurídico juegue un papel fundamental para prevenir, detectar y sancionar el manejo indebido de la deuda. Ello con el fin de evitar la repetición de dramáticas escenas de sobreendeudamiento que han vivido otros países en épocas recientes y nuestro propio país en el último tercio del siglo Xx. La transparencia, el combate a la corrupción y el control de la deuda pública son tareas del presente que permitirán dejar un legado de tranquilidad y bienestar a las generaciones futuras. 


\section{BIBLIOGRAFÍA}

Alonso, José Antonio (2011), "Introducción", en José Antonio Alonso y Carlos Mulas-Granado (eds.), Corrupción, cohesión social y desarrollo: El caso de Iberoamérica, Madrid, Fondo de Cultura Económica, pp. 11-20.

y Carlos Garciamartín (2011), "Causas y consecuencias de la corrupción: una revisión de la literatura”, en José Antonio Alonso y Carlos Mulas-Granado (eds.), Corrupción, cohesión social y desarrollo: El caso de Iberoamérica, Madrid, Fondo de Cultura Económica, pp. 43-72.

Alt, James y David Lassen (2006), "Fiscal Transparency, Political Parties, and Debt in oecd Countries", European Economic Review, 50(6), pp. 14031439.

Arellano, David (2007), "Fallas de transparencia: hacia una incorporación efectiva de políticas de transparencia en las organizaciones públicas", Convergencia, 14(45), pp. 31-46.

Auditoría Superior de la Federación (ASF) (2015), Perfil de México a través de Indicadores Clave, México, Auditoría Superior de la Federación.

Bellver, Ana (2007), "Reformas en materia de transparencia: segunda generación de cambio institucional", Reforma y Democracia, 38, pp. 5-48.

Benito, Bernardino y Francisco Bastida (2009), "Budget Transparency, Fiscal Performance, and Political Turnout: An International Approach", Public Administration Review, 69 (3), pp. 403-417.

Buchanan, James y Richard Musgrave (1999), Public Finance and Public Choice: Two Constrasting Visions of the State, Cambridge, Massachusetts, The MIT Press.

Carpizo, Jorge (2001), "Veintidós años de presidencialismo mexicano: 19782000. Una Recapitulación”, Boletín Mexicano de Derecho Comparado, nueva serie, año XXXIV, núm. 100, enero-abril, pp. 71-99.

Cunill, Nuria (2006), "La transparencia en la gestión pública: ¿Cómo construirle viabilidad?", Estado, gobierno, gestión pública: Revista Chilena de Administración Pública (8), pp. 22-44.

Del Solar, Felipe (2008), “Transparencia, corrupción y acceso a información pública en Chile”, en Andrés Solimano, Vito Tanzi y Felipe del Solar (eds.), Las termitas del Estado, Chile, Fondo de Cultura Económica, pp. 89-144.

Easterly, William (2002), The Elusive Quest for Growth, Cambridge, Massachusetts, The mit Press.

Fox, Jonathan y Libby Haight (2007), "Las reformas a favor de la transparencia: teoría y práctica", Derecho a saber. Balance y perspectivas civicas, pp. 29-64. 
Grimmelikhuijsen, Stephan, Gregory Porumbescu, Boram Hong y Tobin Im (2013), "The Effect of Transparency on Trust in Government: A CrossNational Comparative Experiment", Public Administration Review, 73(4), pp. 575-586.

Héritier, Adrianne (2003), "Composite Democracy in Europe: the Role of Transparency and Access to Information", Journal of European Public Policy, 10(5), pp. 814-833.

Hollyer, James, Peter Rosendorff y Raymond Vreeland (2011), "Democracy and Transparency", The Journal of Politics, 73(04), pp. 1191-1205.

Huntington, Peter (1968), Political Order in Changing Societies, New Haven, Yale University Press.

Kant, Immanuel (2013) [1791], ¿Qué es la ilustración?: y otros escritos de ética, politica y filosofía de la historia, Madrid, Alianza Editorial.

Leff, Haupert (1964), "Economic Development through Bureaucratic Corruption", American Behavioral Scientist, 82(2), pp. 337-341.

Lindstedt, Catherine y Daniel Naurin (2010), "Transparency is not enough: Making Transparency Effective in Reducing Corruption", International Political Science Review, 31(3), pp. 301-322.

Luna, Issa (2013), Movimiento Social del Derecho de Acceso a la Información en México, Instituto de Investigaciones Jurídicas-unam, México.

Merino, Mauricio (2006), "Muchas políticas y un solo derecho", en S. López Ayllón (ed.), Democracia, transparencia y Constitución: propuestas para un debate necesario, México, IIJ-IFAI, pp. 127-156.

Monsiváis, Alejandro (2005), Politicas de transparencia: ciudadania y rendición de cuentas, México, Instituto Federal de Acceso a la Información Pública.

Myrdal, Gunnar (1990), The Political Element in the Development of Economic Theory, EU, New Brunswick, New Jersey.

Ohashi, Hiroshi (2009), "Effects of Transparency in Procurement Practices on Government Expenditure: A Case Study of Municipal Public Works", Review of Industrial Organization, 34(3), pp. 267-285.

Piñar, José Luis (2014), "Transparencia y Derecho de Acceso a la Información pública: algunas reflexiones en torno al derecho de acceso en la Ley 19/2013, de Transparencia, Acceso a la Información y Buen Gobierno", Revista catalana de dret públic, (49), pp. 1-19.

Solimano, Andrés (2008), La corrupción: Motivaciones individuales, fallas del Estado y desarrollo, en Andrés Solimano, Vito Tanzi y Felipe del Solar (eds.), Las termitas del Estado. Ensayos sobre corrupción, transparencia y desarrollo. Chile, Fondo de Cultura Económica, pp. 57-88. 
Tanzi, Vito (2008), "La corrupción y la actividad económica”, en Andrés Solimano, Vito Tanzi y Felipe del Solar (eds.), Las termitas del Estado. Ensayos sobre corrupción, transparencia y desarrollo, Chile, Fondo de Cultura Económica, pp. 23-56.

Uvalle, Ricardo (2008), "Gobernabilidad, transparencia y reconstrucción del Estado", Revista Mexicana de Ciencias Políticas y Sociales, 50 (203).

Weber, Max (2005) [1905], La ética protestante y el espiritu del capitalismo, México, Colofón.

\section{LEGISLACIÓN CONSULTADA}

Constitución Política de los Estados Unidos Mexicanos.

Constituciones y legislación estatal de las 32 entidades federativas en materia de transparencia, deuda pública, fiscalización, responsabilidades de los servidores públicos y códigos penales en Orden Jurídico Nacional, consultado en: <http://www.ordenjuridico.gob.mx/ambest.php> [acceso: marzo de 2016].

Decreto de Reformas y Adiciones al Título Cuarto de la Constitución Política de los Estados Unidos Mexicanos (28/12/82).

Decreto por el que se declaran reformados los artículos 73, 74, 78 y 79 de la Constitución Política de los Estados Unidos Mexicanos (dof 30/07/99).

Decreto por el que se reforman y adicionan diversas disposiciones de la Constitución Política de los Estados Unidos Mexicanos, en materia de transparencia (DOF 07/02/14).

Decreto por el que se reforman y adicionan diversas disposiciones de la Constitución Política de los Estados Unidos Mexicanos, en materia de disciplina financiera de las entidades federativas y los municipios (DOF 26/05/15).

Decreto por el que se reforman, adicionan y derogan diversas disposiciones de la Constitución Política de los Estados Unidos Mexicanos, en materia de combate a la corrupción (DOF 27/05/15).

Ley de Fiscalización y Rendición de Cuentas de la Federación.

Ley Federal de Responsabilidades de los Servidores Públicos.

Ley Federal de Responsabilidades Administrativas de los Servidores Públicos.

Ley Federal de Transparencia y Acceso a la Información Pública Gubernamental.

Ley General de Deuda Pública.

Ley General de Transparencia y Acceso a la Información Pública. 
Proyecto de decreto, por el que se expide la Ley de Disciplina Financiera de las Entidades Federativas y los Municipios; y se reforman, adicionan y derogan diversas disposiciones de las Leyes de Coordinación Fiscal, General de Deuda Pública, y General de Contabilidad Gubernamental (Gaceta Parlamentaria. Cámara de Diputados. 17 de marzo de 2016).

\section{SITIOS DE INTERNET}

ASF <http://www.asf.gob.mx/Section/46_Informes_y_publicaciones>, fecha de consulta: marzo 20 de 2016.

INEGI <http://sc.inegi.org.mx/cobdem/contenido.jsp? rf=false\&solicitud=>, fecha de consulta: marzo 3 de 2016.

SEGOB <http://www.ordenjuridico.gob.mx/>, fecha de consulta: marzo 20 de 2016.

sHCP $<$ http://finanzaspublicas.hacienda.gob.mx/es/Finanzas_Publicas/Estadisticas_Oportunas_de_Finanzas_Publicas $>$, fecha de consulta: marzo 20 de 2016.

Transparencia Mexicana <http://www.tm.org.mx/wp-content/uploads/2013/ 05/01-INCBG-2010-Informe-Ejecutivo1.pdf>, fecha de consulta: marzo 15 de 2016. 